\title{
Universal features of electron-phonon interactions in atomic wires
}

\author{
L. de la Vega ${ }^{1}$, A. Martín-Rodero ${ }^{1}$, N. Agraït $^{2}$ and A. Levy Yeyati ${ }^{1}$ \\ ${ }^{1}$ Departamento de Física Teórica de la Materia Condensada CV and \\ ${ }^{2}$ Departamento de Física de la Materia Condensada CIII, \\ Universidad Autónoma de Madrid, E-28049 Madrid, Spain
}

\begin{abstract}
The effect of electron-phonon interactions in the conductance through metallic atomic wires is theoretically analyzed. The proposed model allows to consider an atomic size region electrically and mechanically coupled to bulk electrodes. We show that under rather general conditions the features due to electron-phonon coupling are described by universal functions of the system transmission coefficients. It is predicted that the reduction of the conductance due to electron-phonon coupling which is observed close to perfect transmission should evolve into an enhancement at low transmission. This crossover can be understood in a transparent way as arising from the competition between elastic and inelastic processes.
\end{abstract}

PACS numbers: 73.23.-b, 72.10.Di, 73.63.Nm

\section{INTRODUCTION}

Metallic nanowires, including atomic contacts and atomic chains have become ideal systems for testing predictions of quantum transport theory [1]. Many transport properties in the normal state like shot-noise 2], conductance fluctuations [3] and others in the superconducting state [4] have been measured with a remarkable agreement with the theoretical predictions [1]. A key ingredient in this analysis is provided by the set of transmission coefficients $\tau_{n}$ (the so-called mesoscopic PINcode), in terms of which a generic transport property $F(V)$, where $V$ is the bias voltage, can be written as $F(V)=\sum_{n} f\left(V, \tau_{n}\right), f(V, \tau)$ being the contribution of a single conduction channel [4]. The validity of this decomposition relies on the usually large difference in energy scales between the electronic excitations involved, $\sim e V$, and the typical scale $W_{e l}$ for the variation of the normal density of states. This one-electron description applies as far as charging effects can be neglected due to the strong coupling of the electronic states of the atomic region with the metallic electrodes.

Recently there has been much interest in the formation of metallic atomic chains using STM and break junction techniques [5] allowing the possibility to study electron transport through these nearly ideal one-dimensional systems. In particular, experiments in Au chains show unambiguously the presence of features associated to a quasi one-dimensional phonon spectrum $[$ ] . The experimental conductance exhibits tiny steps at voltages corresponding to vibrational modes of the finite suspended chain. A number of theoretical papers have addressed the explanation of these basic features 7, 8, 9]. It could be interesting to investigate whether the type of description commented above in terms of the system transmission coefficients could be valid to analyze the effects of electron-phonon (EPH) interactions, which is reasonable as the relevant phonon energies $\hbar \omega \sim 10 \mathrm{meV}$ are still small compared to the typical $W_{e l} \sim 0.1-1 \mathrm{eV}$ in metal-

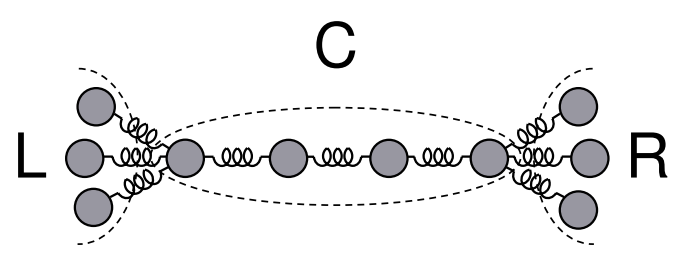

FIG. 1: Schematic representation of an atomic wire suspended between metallic electrodes. For the theoretical description the system is decomposed into $\mathrm{L}$ (left), $\mathrm{R}$ (right) and $\mathrm{C}$ (central) regions. The labels 1 and $N$ denote the outermost sites of the wire.

lic atomic conductors [10]. Starting from a microscopic model and performing reasonable simplifying assumptions we show in this work that the main features due to EPH coupling in a single-channel conductor can be described by a universal function of its transmission $\tau$. The sign of the conductance correction, which is negative for large transmission, evolves into a positive correction for low transmission. This behavior can be understood as a competition between elastic and inelastic processes. We show that these predictions apply naturally to the case of atomic chains of arbitrary length.

\section{THEORETICAL MODEL}

As a starting point we consider a tight-binding model for describing the electronic properties which is well adapted to spatially inhomogeneous systems like atomic size conductors 10, 11]. This approach can be straightforwardly extended to incorporate the phonon degrees of freedom [7]. We consider the type of geometry schematically depicted in Fig. 1 consisting of a left and right electrode electrically and mechanically coupled to a central atomic size region. The model allows in principle to incorporate not only the phonons of the isolated central region (as done in most previous works [7, 8, 9 ]) 
but also to take into account the effect of the electrodes in their dynamics. Within this approach the electronic Hamiltonian can be written as $\hat{H}_{e l}=\sum_{i j, \sigma} h_{i j} \hat{c}_{i \sigma}^{\dagger} \hat{c}_{j \sigma}$, where $\hat{c}_{i \sigma}^{\dagger}$ creates an electron in site $i$ with spin $\sigma(i, j$ run over sites of the whole system); and equivalently for the phonon degrees of freedom we have $\hat{H}_{p h}=\sum_{i} \hat{p}_{i}^{2} / 2 M+$ $\sum_{i j} \hat{u}_{i} A_{i j} \hat{u}_{j}$, where the atomic displacements $\hat{u}_{i}$ and the corresponding momenta $\hat{p}_{i}$ satisfy the quantization relation $\left[\hat{u}_{j}, \hat{p}_{k}\right]=i \hbar \delta_{j k}$. Both the electronic Hamiltonian and the dynamical matrix $\mathbf{A}$ can be decomposed as $\hat{H}=\hat{H}_{L}+\hat{H}_{R}+\hat{H}_{C}+\hat{V}_{L C}+\hat{V}_{C R}$, describing respectively the isolated $L, R$ and $C$ regions and the coupling between the central region and the electrodes. Finally, the EPH coupling $\hat{H}_{e-p h}=\sum_{i j} \lambda_{i j}\left(\hat{u}_{i}-\hat{u}_{j}\right) \hat{c}_{j}^{\dagger} \hat{c}_{i}$ is obtained by expanding the electronic Hamiltonian to first order in the lattice displacements 7]. We shall consider the simplest version of this tight-binding model where only the hopping terms between neighboring sites are non-zero and thus the EPH coupling constants $\left|\lambda_{i j}\right|=\partial h_{i j} / \partial r$ are non-local and short ranged.

The transport properties of this model are conveniently calculated in terms of non-equilibrium Green function techniques. Using the electronic propagators in Keldysh space $G_{i j}^{\alpha \beta}\left(t, t^{\prime}\right)$, where $\alpha, \beta \equiv+,-$ denote the two branches of the Keldysh contour 12], the current between two sites $i, j$ can be written as

$$
I_{i j}=\frac{2 e}{\hbar} h_{i j}\left(G_{i j}^{+-}(t, t)-G_{j i}^{+-}(t, t)\right) .
$$

The EPH interaction is included to second order in perturbation theory, which is appropriate in the metallic case [13]. The corresponding self-energy insertions can be written in terms of the unperturbed propagators as

$$
\Sigma_{i j}^{\alpha \beta}(\omega)=i \alpha \beta \int \frac{d \omega^{\prime}}{2 \pi} \sum_{k l} \lambda_{i k} \lambda_{l j} \bar{D}_{i k, l j}^{0, \alpha \beta}\left(\omega-\omega^{\prime}\right) G_{k l}^{0, \alpha \beta}\left(\omega^{\prime}\right),
$$

where $\bar{D}_{i k, l j}^{0, \alpha \beta}=D_{i l}^{0, \alpha \beta}-D_{i j}^{0, \alpha \beta}-D_{k l}^{0, \alpha \beta}+D_{k j}^{0, \alpha \beta}$, with $D_{i j}^{0, \alpha \beta}$ denoting the phonon propagators. If the phonons are assumed to be in thermal equilibrium [14], the frequency dependent propagators in Keldysh space are related to the advanced and retarded ones by $D_{i j}^{0, \alpha \beta}=$ $\left(n_{B}+\delta_{\beta,+}\right) D_{i j}^{0, r}-\left(n_{B}+\delta_{\alpha,-}\right) D_{i j}^{0, a}$, where $n_{B}$ is the Bose-Einstein distribution. On the other hand, the retarded and advanced propagators are formally given by $\mathbf{D}^{0, r, a}(\omega)=\left[(\omega \pm i \eta)^{2}-\mathbf{A}\right]^{-1}$ and can be evaluated using similar techniques as for their electronic counterparts [16]. Up to this level of approximation, the correction to the current due to EPH coupling in the chain geometry of Fig. 11 can be decomposed into elastic and inelastic contributions, ie. $\delta I=\delta I_{e l}+\delta I_{i n}$ [15], where

$$
\begin{aligned}
\delta I_{e l}= & \frac{16 e}{h} \Gamma_{L} \Gamma_{R} \int d \omega \sum_{i j} \operatorname{Re}\left(G_{1 i}^{0, r} \Sigma_{i j}^{r} G_{j N}^{0, r} G_{N 1}^{0, a}\right)\left(f_{L}(\omega)-f_{R}(\omega)\right) \\
\delta I_{i n}= & -\frac{8 e i}{h} \Gamma_{L} \Gamma_{R} \int d \omega \int \frac{d \omega^{\prime}}{2 \pi} \sum_{i j k l} \lambda_{i k} \lambda_{l j} G_{1 i}^{0, r}(\omega) G_{k N}^{0, r}\left(\omega^{\prime}\right) G_{N l}^{0, a}\left(\omega^{\prime}\right) G_{j 1}^{0, a}(\omega) \\
& \times\left[\bar{D}_{i k, l j}^{0,-+}\left(\omega-\omega^{\prime}\right) f_{L}(\omega)\left(1-f_{R}\left(\omega^{\prime}\right)\right)-\bar{D}_{i k, l j}^{0,+-}\left(\omega-\omega^{\prime}\right) f_{R}\left(\omega^{\prime}\right)\left(1-f_{L}(\omega)\right)\right],
\end{aligned}
$$

$\Gamma_{L, R}$ and $f_{L, R}$ being the tunneling rates coupling the chain to the leads (assumed to be energy independent) and the corresponding Fermi distributions. Within this decomposition the inelastic contribution corresponds to the transfer of an electron with the real emission (or absorption at finite temperature) of a phonon mode; while in the elastic process the interaction with phonons induces a transition into an intermediate virtual state resulting in a renormalization of the transmission.

\section{RESULTS}

Let us first analyze the effect of EPH interactions in the conductance for the ideal chain geometry. The main features of this case at zero temperature are illustrated in Fig. 2 The ratio between the first neighbors EPH coupling constant $\lambda$ and the hopping term $t$ within the chain is taken to be $7 \times 10^{-3}$ while the maximum phonon mode of the uncoupled chain is taken as $\hbar \omega_{N} \sim 0.03 t$ (i.e. $\left.\hbar \omega_{N} / t \ll 1\right)$ in order to reproduce the typical features observed experimentally for Au chains [6]. The comparison with the experimental results is illustrated in Fig. 3] which will be discussed below. As can be observed in Fig. 2] the conductance exhibits small steps which 


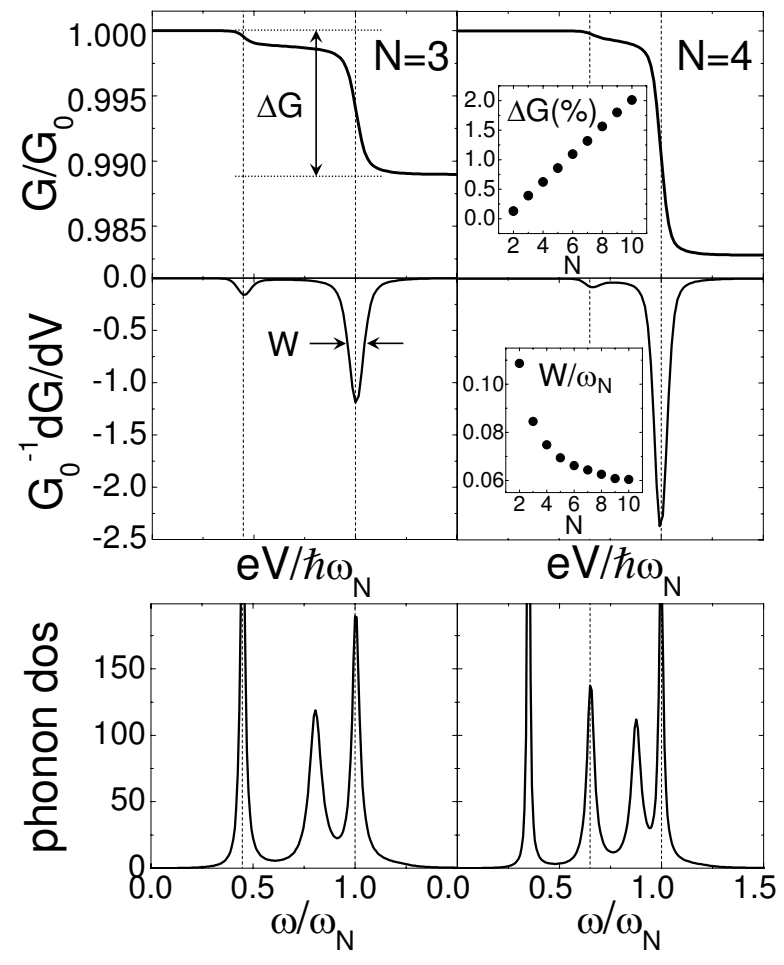

FIG. 2: Features due to EPH coupling in the conductance for the ideal chain geometry containing 3 and 4 atoms. The insets in the right upper and middle panels show the scaling of the total conductance step $\Delta G$ and its width $W$ with the number of atoms $N$. The correlation of these features with the phonon spectrum is illustrated by the included average phonon density of states within the chain in the lower panels. $G_{0}$ denotes the quantum of conductance $2 e^{2} / h$.

can be associated with the onset of inelastic emission of longitudinal phonons. The more pronounced structure is associated with the highest phonon mode of the chain but smaller features related to lower modes also appear. In this zero temperature limit it is possible to correlate the width of the steps with the corresponding width of the resonances observed in the phonon density of states of the chain (see lower panels of Fig. 2). This width is in turn controlled by the mechanical coupling of the chain with the bulk electrodes, thus providing and intrinsic width of the steps in the conductance 17. Notice that only steps associated with phonon modes having a definite parity (even or odd) are observed for a given number of atoms in the chain. The total step size is found to follow simple scaling laws with the EPH coupling $\lambda$ and the highest phonon frequency of the chain $\omega_{N}$, i.e $\delta G \sim \lambda^{2} / \omega_{N}$, while it increases roughly linearly with $N$ as illustrated in the upper right inset of Fig. 2] in agreement with the experimental observations [ 6 . Physically, this behavior arises from the increase of the interaction probability with increasing chain length and from the increase of the vibration amplitude for decreasing frequency. The width of the step is also found to scale simply as $\sim 1 / N$ (see inset

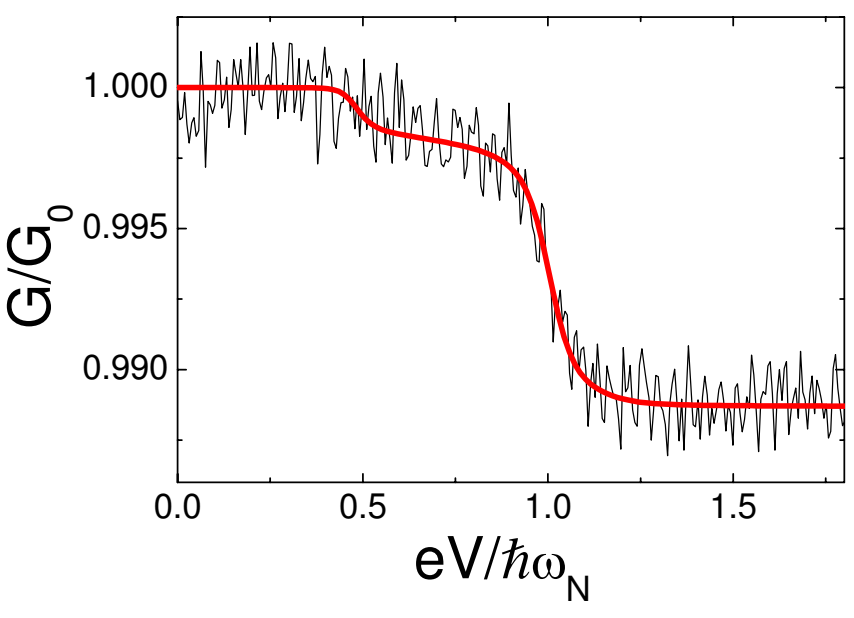

FIG. 3: (color online) Comparison between the full model calculations and the experimental measurements for the conductance of $\mathrm{Au}$ atomic chains. The experimental data correspond to a typical curve from the experiments described in Ref. [6], while the theoretical curve has been obtained for the case $N=3$ using the set of parameters discussed in the text.

in right middle panel of Fig. 21). As can be observed in Fig 3 there is a good agreement between our model calculations and the typical experimental results of Ref. [6]. The theoretical curve corresponds to a short chain $(N=3)$, and exhibits a small step at low bias in addition to the main conductance step. In spite of the experimental noise this smaller feature can also be distinguished in the experimental curve.

We have also analyzed deviations from the ideal chain geometry, in particular we have studied the effect of a zig-zag configuration, taking into account the appearance of transversal modes in the chain. We have found that these effects do not modify substantially the overall behavior discussed above. The robustness of these results and the simplicity of the scaling behavior found for the linear chain suggest that the theory could be reduced to a minimal model keeping only the essential ingredients. As we show below this is still the case when the bare channel transmission is reduced with respect to one. Thus, assuming that the typical phonon energies are small on the scale $W_{e l}$, the energy dependence of the unperturbed electron propagators can be neglected. One can also relate these propagators to the scattering properties of the system at the Fermi level by means of the relations [18]

$$
\hat{s}=\left(\begin{array}{cc}
r_{L} & t_{L R} \\
t_{R L} & r_{R}
\end{array}\right)=\mathbf{I}-2 i\left(\begin{array}{cc}
\Gamma_{L} G_{11}^{0, r} & \sqrt{\Gamma_{L} \Gamma_{R}} G_{1 N}^{0, r} \\
\sqrt{\Gamma_{L} \Gamma_{R}} G_{N 1}^{0, r} & \Gamma_{R} G_{N N}^{0, r}
\end{array}\right) .
$$

These coefficients are related to the normal transmission $\tau$ by $\tau=\left|t_{L R}\right|^{2}=\left|t_{R L}\right|^{2}=1-\left|r_{L, R}\right|^{2}$. Notice that the transmission can be reduced either due to asymmetries in the coupling to the electrodes or to the presence of an impurity atom within the chain. On the other hand, 


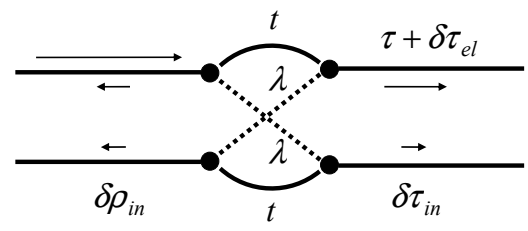

FIG. 4: Schematic representation of a minimal model accounting for the transition between negative and positive conductance step as a function of the transmission based in the mapping proposed in Refs. [20]. A single phonon mode between two sites is considered. The two channels correspond to the absence or to the presence of an excited phonon. The arrows indicate all possible paths for an incident electron: elastic and inelastic transmission and reflection.

if one is interested in the total size of the steps and not in their width, the expressions can be greatly simplified by taking the limit of negligible broadening of the chain phonon states while maintaining their thermal population. In this limit it is possible to factorize the corrections to the current into a purely electronic factor and another one associated with the coupling to the phonon system. Up to a uniform background correction $\delta G_{b}$, which does not exhibit structure at the phonon frequencies, one obtains from Eqs. (314) simple analytical expressions for the conductance which for a half-filled band and at zero temperature reduce to

$$
\begin{gathered}
\delta G_{e l}-\delta G_{b}=-\frac{2 e^{2}}{h} \tau^{2} \frac{\lambda^{2}}{t^{2}} \sum_{r=1}^{N} F_{e p h, r} \theta\left(|e V|-\hbar \omega_{r}\right) \\
\delta G_{i n}=\frac{2 e^{2}}{h} \tau(1-\tau) \frac{\lambda^{2}}{t^{2}} \sum_{r=1}^{N} F_{e p h, r} \theta\left(|e V|-\hbar \omega_{r}\right),
\end{gathered}
$$

where $F_{e p h, r}=\left|\sum_{j=1}^{N-1}(-1)^{j}\left(a_{j, r}-a_{j+1, r}\right)\right|^{2}$, with $a_{j, r}$ denoting the amplitude of the chain atom at site $j$ in the mode of frequency $\omega_{r}$ (normalization $\sum_{j=1}^{N} a_{j, r}^{2}=$ $\left.\hbar / M \omega_{r}\right)$. It is interesting to observe that these two contributions are universal functions of the transmission through the chain. Notice that the inelastic contribution is always positive while the elastic one yields always a reduction of the total current. On the other hand, while the elastic contribution increases monotonously with $\tau$, the inelastic correction behaves as $\tau(1-\tau)$ and thus vanishes in the limit of perfect transmission as in the case of shot noise in a quantum point contact 19. As a result of the competition between the elastic and the inelastic parts, the total correction exhibits a change of sign as a function of the transmission. In this simplified model the step in the current at the phonon frequencies scales as $\tau(1-2 \tau)$, i.e. the change in the sign of the correction occurs for $\tau=1 / 2$. This type of behavior can also be qualitatively obtained by means of a mapping of the EPH problem into a coherent multichannel scattering model, as proposed by several authors 20]. For the simplest case of a single localized mode between two atoms in which

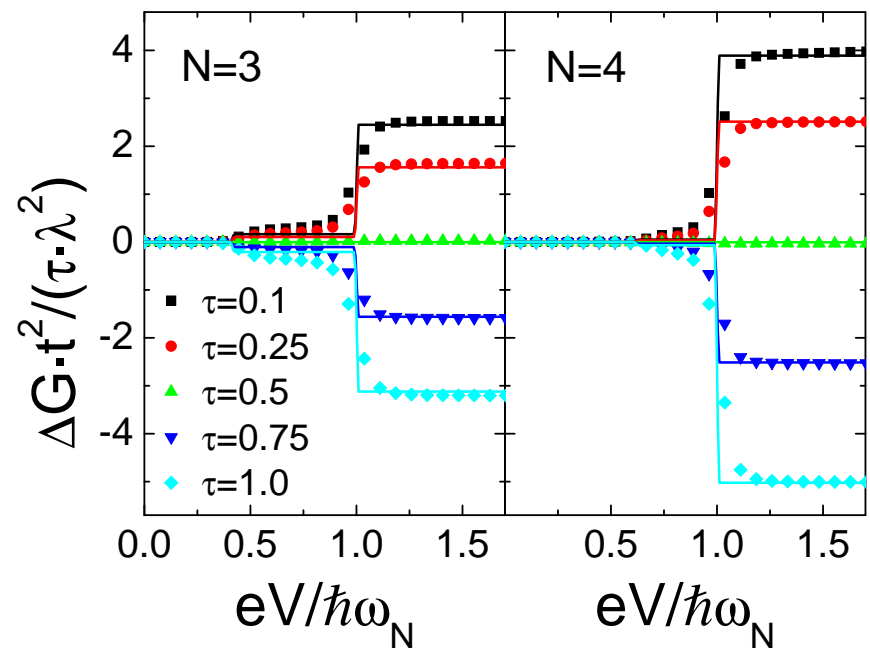

FIG. 5: (color online) Evolution of the conductance steps as a function of the transmission for an atomic chain with $N=3$ and 4 . The symbols correspond to the full calculation while the full lines were obtained using Eqs. 6 6 7).

an incoming electron can be transmitted either elastically or inelastically, as schematically depicted in Fig. 4. this model would predict $\delta \tau_{e l} \approx-(\lambda / t)^{2} \tau(1-r)(1+2 r)$, where $r=\left|r_{L, R}\right|=\sqrt{1-\tau}$, and $\delta \tau_{\text {in }} \approx(\lambda / t)^{2} \tau(1-\tau)$, i.e. a change in the sign of the correction for $\tau \sim 0.41$; while the inelastic reflection is given by $\delta \rho_{i n}=(\lambda / t)^{2} \tau^{2}$. Thus in the $\tau \rightarrow 1(\tau \rightarrow 0)$ limit the inelastic transitions correspond mainly to back (forward) scattering. Although this extremely simple model accounts for the overall behavior, the absence of a correct inclusion of the restrictions imposed by the Pauli principle is responsible for the discrepancies with the correct result (Eqs. (617)). It is worth noticing that the factor $F_{e p h, r}$ in Eqs. (617) shows explicitly the selection rules of modes with a definite parity. For the highest frequency mode one has $a_{i, N} \approx-a_{i+1, N}$ which produces a maximum size for the corresponding step. It is also straightforward to show that the size of this step increases roughly linearly with $N$. The evolution of the conductance steps as a function of the transmission predicted by Eqs. (67) is compared in Fig. 5 with the results of the full calculation. As can be noticed the behavior of the full calculation is reproduced except for the smearing of the steps due to the intrinsic width of the phonon modes.

We have also analyzed in detail the validity of the simplified model leading to Eqs. (617) for significant deviations with respect to the half-filled band case. One obtains corrections to these equations which are of order $\left(\epsilon / W_{e l}\right)^{2}$, where $\epsilon$ measures the shift of the band with respect to half-filling. We have checked numerically that these corrections become of importance only in the limit $\left(\epsilon / W_{e l}\right) \sim 1$, i.e. close to the nearly empty or full cases. 


\section{CONCLUSIONS}

We have presented a theoretical analysis of EPH interactions in atomic size conductors. Our description allows to incorporate in an equal footing the effects due to electrical and mechanical coupling with the electrodes. We have shown that in the typical conditions corresponding to metallic atomic chains the main features of EPH interactions can be accounted for by simple analytical expressions factorized into a purely electronic part and another describing the EPH coupling. While the electronic part can be written in terms of the bare transmission, the phonon part accounts for the selection rules and the dependence on the length observed for the case of an ideal chain. A prediction which deserves further experimental investigation concerns the inversion of the conductance steps for a single channel at $\tau \sim 1 / 2$. Atomic Au chains containing $\mathrm{S}$ or $\mathrm{O}$ impurities which can reduce substantially its transmission as well as atomic contacts exhibiting partially open channels like for $\mathrm{Al}[\underline{10}$ are promising candidates to test these predictions.

Note added: while completing this manuscript we became aware of related work by M. Paulsson et al. (cond-mat/0505473) and J.K. Viljas et al.(cond-mat/0508470). The authors would like to thank financial support from Spanish MEC under contracts BMF2001-0150 and MAT2004-03069, and MAT2002$11982 \mathrm{E}$ through the SONS program of the ESF which is also supported by the EC, 6th Framework Program. Fruitful discussions with J.C. Cuevas and J.M. van Ruitenbeek are acknowledged.

[1] N. Agraït, A.L. Yeyati and J.M. van Ruitenbeek, Phys. Rep. 377, 81 (2003).
[2] R. Cron et al. Phys. Rev. Lett. 86, 4104 (2001).

[3] B. Ludoph et al. Phys. Rev. B 61, 8561 (2000).

[4] E. Scheer et al., Phys. Rev. Lett. 78, 3535 (1997); E. Scheer et al., Nature 394, 154 (1998); M. Goffman et al., Phys. Rev. Lett. 85, 170 (2000).

[5] A.I. Yanson et al., Nature 395, 783 (1998).

[6] N. Agraït et al. Phys. Rev. Lett. 88, 216803 (2002); N. Agrait et al. Chem. Phys. 281, 231 (2002).

[7] M.J. Montgomery et al. J. Phys.: Condens. Matter 15, 731 (2003).

[8] T. Frederiksen et al. Phys. Rev. Lett. 93, 256601 (2004).

[9] L. Stella et al. Surf. Science 566, 430 (2004).

[10] J.C. Cuevas, A.L. Yeyati and A. Martín-Rodero, Phys. Rev. Lett. 80, 1066 (1998); L. de la Vega et al. Phys. Rev. B 70, 113107 (2004).

[11] M. Brandbyge et al., Phys. Rev. B 60. 17064 (1999); T.N. Todorov et al., Phil. Mag. B 80, 421 (2000).

[12] L.V. Keldysh, Sov. Phys. JETP 20, 1018 (1965).

[13] A.B. Migdal, Sov. Phys. JETP 1, 996 (1958).

[14] This is consistent with the assumption of good coupling with the bulk phonons. Deviations with respect to the equilibrium distribution are not relevant for the present discussion and will be analyzed elsewhere.

[15] C. Caroli et al., J. Phys. C 5, 21 (1972).

[16] E.N. Economou Green's Functions in Quantum Physics, Springer-Verlag (1983).

[17] Other possible mechanisms providing an intrinsic width of the phonon spectrum like the emission of electron-hole pairs are expected to give a much smaller contribution.

[18] S. Datta Electronic Transport in Mesoscopic Systems, Cambridge University Press (1997).

[19] V.A. Khlus, Sov. Phys. JETP 66, 1243 (1987); M. Büttiker, Phys. Rev. B 46, 12485 (1992).

[20] J. Bonca and S. Trugman, Phys. Rev. Lett. 75, 2566 (1995); H. Ness et al. Phys. Rev. B 63, 125422 (2001); L.E.F. Foa-Torres et al., Phys. Rev. B 64, 193304 (2001). 